\title{
Localization and Quantification of Total UV Absorbing Compounds in Green Ash (Fraxinus pennsylvanica)
}

\author{
Vanessa Ferchaud ${ }^{1}$, Yadong $\mathrm{Qi}^{2}$ and Kit Chin $^{1}$
}

${ }^{1}$ Southern University Agricultural, Research and Extension Center, Baton Rouge, Louisiana, United States, ${ }^{2}$ Southern University and A\&M College, Baton Rouge, Louisiana, United States

The sun emits three different types of UV radiation: UVA (400-315 nm), UVB (315-280 nm) and UVC $(280-100 \mathrm{~nm})$. The entire UVC and most of the UVB radiation is absorbed by the stratospheric ozone layer, which provides instant protection to all life forms the earth. However, in recent decades, enhanced UVB radiation has been observed on the ground level due to the stratospheric ozone depletion caused by man-made activities. Despite the global ban of certain ozone-depleting chemicals such as CFCs, the temporal and spatial recovery of the ozone layer is uncertain. Forests are accounted for the majority of living biomass on earth; thus, it is important to understand how diverse forest tree species cope with the harmful UVB radiation as it has a disproportionately large photobiological effect on forest tree species [1].

Significant progress has been made in recent years toward an understanding of the UV tolerance mechanism in diverse southern broadleaf tree species in the USA $[2,3,4,5,6,7,8]$. The UV tolerance ability depends on species and their growing conditions and involves multicomplex interactions among biophysical, biochemical, anatomical and genetic processes. The biophysical properties deal with leaf reflectance, transmittance, and absorbance, depth of light penetration, and epidermal transmittance to UVB radiation. The anatomical aspect relates to leaf development, surface morphology, transverse profile, and internal ultrastructure. The biochemical aspect is associated with localization, quantification and qualification of leaf UV-B absorbing compounds (phenolics and flavonoids), and the genetic process involves DNA damage/repair and UVR gene expressions.

Green Ash (Fraxinus pennsylvania) is the most widely distributed ash in the USA, planted as urban trees, windbreaks, and landscape specimens. The aim of this study was to localize, visualize, and quantify UV absorbing compounds in young and mature green ash leaves. The goal is to gain biochemical and anatomical insight into the UV tolerance for this species.

Leaf samples of green ash were collected from sun-exposed portions at the terminal end, $10-50 \mathrm{~cm}$ of a branch, from five individual green ash trees in spring (April) and summer (August) during a growing season in 2009 on Southern University and A\&M College campus in Baton Rouge, LA., USA.

Visualization of UV-Absorbing Compounds - The fresh leaves were sectioned into approximately $0.5 \mathrm{~mm} \times 0.5 \mathrm{~mm}$ squares pieces using a sharp razor and embedded on a specimen disc using OTCcompound (Jung Tissue Freezing Medium, Leica Microsystems) and placed in the chamber of a cryostat (Leica CM-1850 UV Microtome, Nussloch, Germany) for sectioning at $40 \mu \mathrm{m}$ thickness. Rapid freezing at a low temperature of $-20^{\circ} \mathrm{C}$ was utilized depending on leaf moisture content. One drop of $0.2 \%$ Naturstoffreagenz-A (NA) (from Sigma Aldrich, St. Louis, MO) [0.2\%, w/v, $0.2 \mathrm{~g}$ amino ethyl diphenyl boric acid in $100 \mathrm{ml}$ distilled water stock solution] was added to a slide and then covered with a glass coverslip. One drop of phosphate buffer (from Sigma Aldrich, St. Louis, MO) [34 mmol/L, pH 6.6 $@ 25^{\circ} \mathrm{C}$ ] was added to the slide and covered with a coverslip. Images were taken using the monochrome camera and bright-field colour camera with Leica DMI6000 B Inverted Research Automated Robotic Fluorescent Imaging Microscope. 
Spectrophotometric Quantification of UV-Absorbing Compounds - Three (3) sun-exposed leaves were randomly selected and one (1) round disc approximately $6 \mathrm{~mm}$ was punched from each leaf in the middle of the lamina away from the midrib. The discs were placed in a $1.5-\mathrm{mL}$ microfuge tube and homogenized with a Teflon pestle using liquid nitrogen. One $\mathrm{mL}$ of acidified methanol $\left(\mathrm{CH}_{3} \mathrm{OH} ; \mathrm{H}_{2} \mathrm{O}\right.$ : $\mathrm{HCl}, 79: 20: 1$, v/v) was then added to the 1.5-mL microfuge with the powdered disc and mixed homogeneously. Spectra of the extracts $(190-700 \mathrm{~nm})$ were obtained with a UV/Visible Genesys 6 Spectrophotometer.

The NA-stained green ash spring sampled leaves showed wall-bound UV-absorbing compounds readily visible in the epidermal layers and nearby palisades tissues and were rendered very bright and visible under the monochrome camera (Fig. 1. a, b). Fig. 1c shows a bright field colour image of the green ash leaf petiole with 1 drop phosphate buffer.

The UV absorbing compounds in the summer sampled green ash leaves (Fig. 1. d, e) were localized and presented primarily in leaf petiole vascular bundle and leaf epidermal layers, as well as scattered through the palisades and sparsely visible in the vascular bundles. Fig. 1f is a colour bright field image with 1 drop of phosphate buffer. The leaf trichomes (leaf hairs) were more distinct containing UV absorbing compounds (Fig. 1d).

Comparing the total UV absorbing compound absorbance profile (Fig. 2) between the spring (April) and summer (August) leaves, the summer leaves showed the higher value in UVC region, but there was no significant difference in the UVB region (280-315nm). This indicates that the spring leaves were just as efficient as the summer leaves in defence of harmful UVB radiation.

The leaves of green ash generally ranked low in the amount of total UV-absorbing compounds compared to the other broadleaf tree species; however, the green ash leaves had the ability to absorb $95 \%$ of UVB at 300nm radiation and this absorption was mainly taken place in the leaf upper epidermal layer within the thickness less than 20um [6]. The epidermal function of UVB screening effectiveness was considered the main characteristics of UV tolerance in green ash. This study proves that the remarkable presence of UV absorbing compounds in the upper and lower leaf epidermal layers in both young and mature leaves is directly responsible for the UVB absorption, contributing to the UVB screening effectiveness in the leaves of green ash. Thus, the strategic location and accumulation of UV absorbing compounds in leaves function as an adaptive mechanism in plant self-defence against the harmful UVB radiation [9]. 


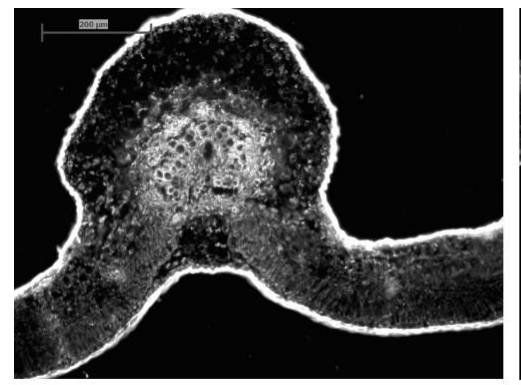

a

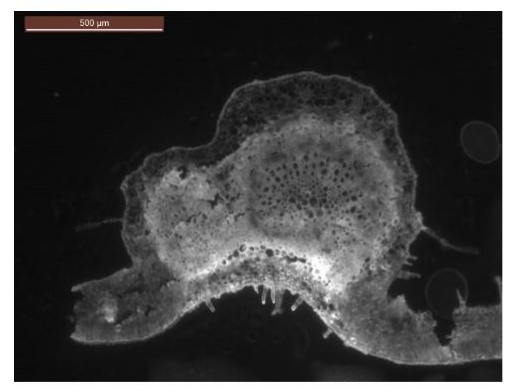

d

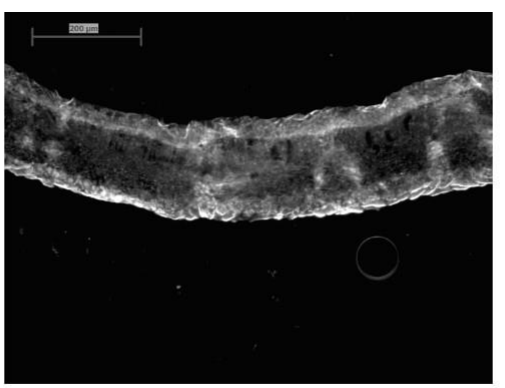

b

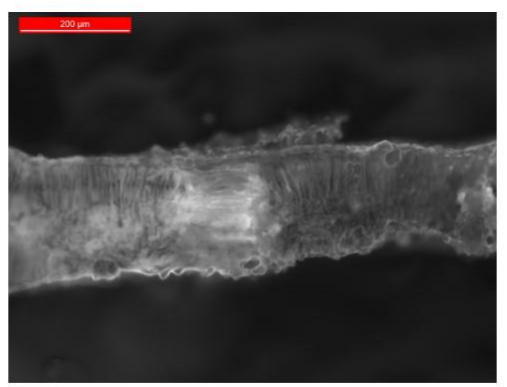

e

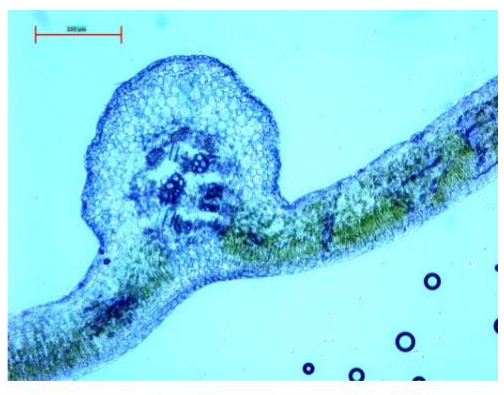

C

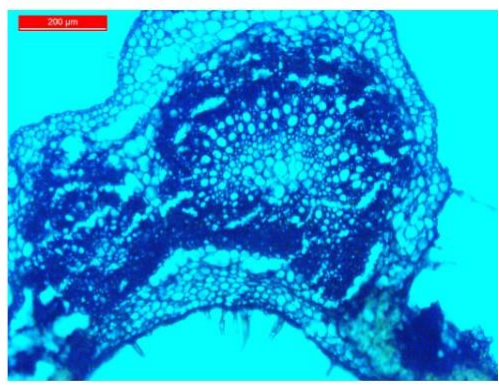

$f$

Figure 1. Localization and visualization of UV absorbing compounds in green ash leaves. a. Young leaf petiole cross-section using GFP-NA stain taken with a monochrome camera. b. Young leaf cross-section using GFP-NA stain taken with a monochrome camera. c. Young leaf petiole cross-section with a brightfield camera and 1 drop phosphate buffer. d. Mature leaf petiole cross-section using GFP-NA stain taken with monochrome camera. e. Mature leaf cross-section using GFP-NA stain taken with monochrome camera. f. Mature leaf petiole cross-section with a bright-field camera and 1 drop of phosphate buffer.

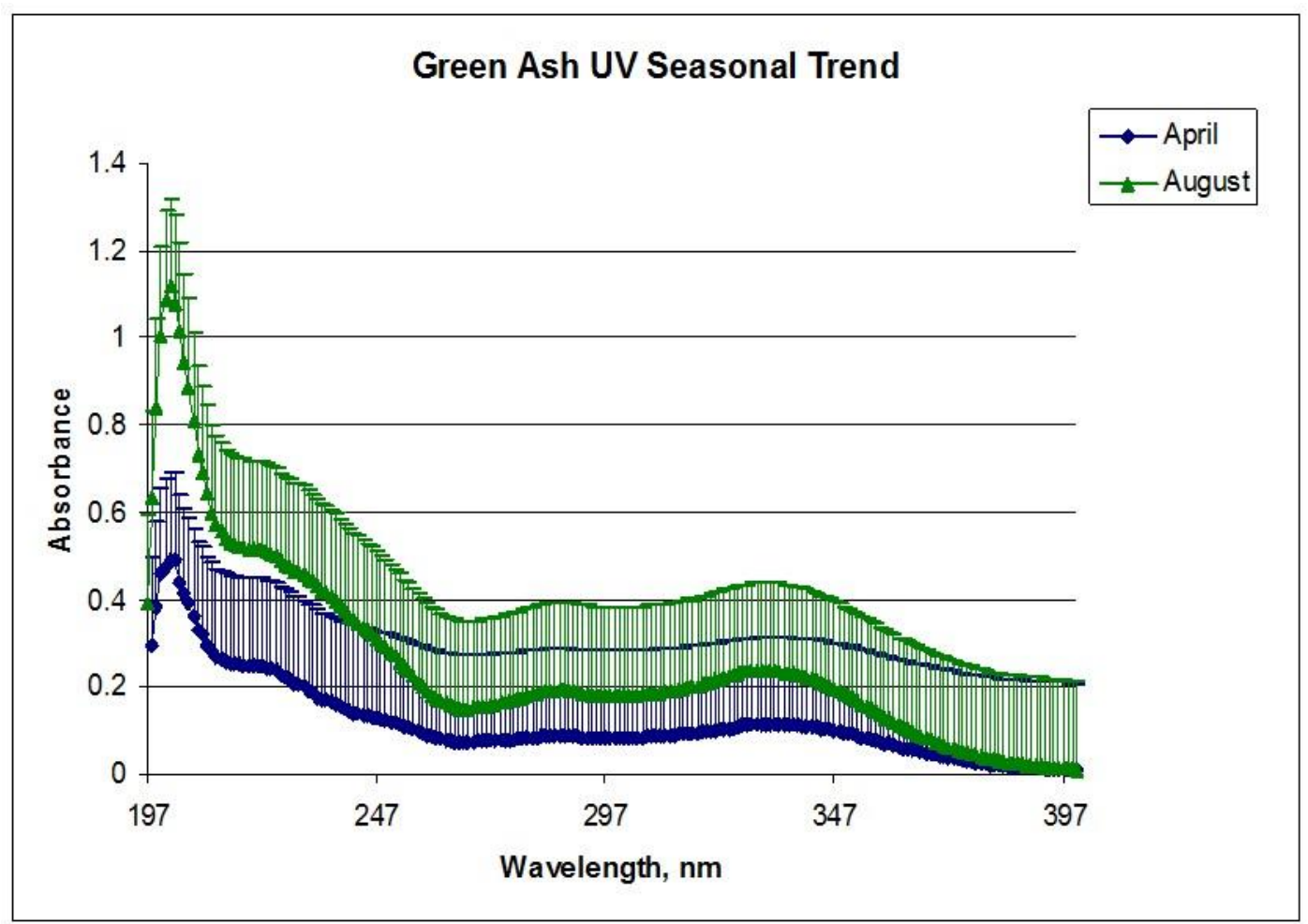

Figure 2. Green ash leaf total UV (197-400 nm) absorbance profile with Mean+1SD, $(\mathrm{n}=5)$. 


\section{References}

[1] Singh, S. S., P. Kumar, and A. K. Rai. 2006. Ultraviolet Radiation Stress: Molecular and Physiological Adaptations in Trees, DOI: 10.1007/1-4020-4389-9_6. p91-110. In: Abiotic Stress Tolerance in Plants, Springer.

[2] Qi, Y., S. Bai, T. C. Vogelmann, G. Heisler, and J. Qin, 2002, Methodology for a comprehensive evaluation of UV-B tolerance in trees. Proc. of SPIE, 4482:367-380.

[3] Qi, Y. S. Bai, W. Gao, and G. M. Heisler. 2003a. Intra and inter-specific comparisons of UV-B absorbing compound concentrations in southern broadleaf trees in the USA. Proc. of SPIE, 4896:120-129. [4] Qi, Yadong; Bai, Shuju; Heisler, Gordon M. 2003b. Changes in ultraviolet-B and visible optical properties and absorbing pigment concentrations in pecan leaves during a growing season. Agricultural and Forest Meteorology, 120(1-4):229-240.

[5] Qi, Y., S. Bai, T.C. Vogelmann, and G. M. Heisler. 2004. Penetration of UV-A, UV-B, blue and red light into leaf tissues of pecan measured by a fibre-optic microprobe system. Proc. of SPIE, 5156:291300 .

[6] Qi. Y., G. M. Heisler, W. Gao, T. C. Vogelmann, and S. Bai. 2010. Characteristics of UV-B radiation tolerance of broadleaf trees in Southern USA, Chapter 21: p509-530. In: UV Radiation in Global Change: Measurements, Modeling and Effects on Ecosystem, Springer-Verlag and Tsinghua University Press. 550 p. ISBN: 978-3-642-03312-4.

[7] Vanessa A. Ferchaud, Yadong Qi and Kit L. Chin. 2016. Localization of UV Absorbing Compounds in Nuttall Oak (Quercus nuttallii) Leaves Using Naturstoffreagenz-A (NA) and the Leica DMI6000 B Inverted Robotic Microscope. Microscopy \& Microanalysis 22(Suppl 3): 1204-1205.

[8] Vanessa A. Ferchaud, Yadong Qi, and Kit L. Chin. 2018. Localization and Quantification of Total UV Absorbing Compounds in Chinese Elm (Ulmus parvifolia). Microscopy and Microanalysis 24(S1):11941195, DOI: $10.1017 /$ S1431927618006451

[9] The authors acknowledge funding from USDA-NIFA Grant 2014-38821-22415. 